\title{
Erlotinib-based doublet targeted therapy versus erlotinib alone in previously treated advanced non-small-cell lung cancer: a meta-analysis from 24 randomized controlled trials
}

\author{
Jian-Wei Gao ${ }^{1, *}$, Ping Zhan ${ }^{1, *}$, Xiang-Yu Qiu ${ }^{2}$, Jia-Jia Jin ${ }^{1}$, Tang-Feng Lv ${ }^{1}$ and Yong \\ Song ${ }^{1}$ \\ ${ }^{1}$ Department of Respiratory Medicine, Jinling Hospital, Nanjing University School of Medicine, Nanjing, China \\ 2 The Research Institute of General Surgery, Jinling Hospital, Nanjing University School of Medicine, Nanjing, China \\ * These authors have contributed equally to this study \\ Correspondence to: Tang-feng Lv, email: bairoushui@163.com \\ Yong Song, email: yong_song6310@yahoo.com
}

Keywords: erlotinib, targeted therapy, advanced non-small cell lung cancer, meta-analysis

Received: November 22, $2016 \quad$ Accepted: March 15, $2017 \quad$ Published: May 31, 2017

Copyright: Gao et al. This is an open-access article distributed under the terms of the Creative Commons Attribution License 3.0 (CC BY 3.0), which permits unrestricted use, distribution, and reproduction in any medium, provided the original author and source are credited.

\section{ABSTRACT}

Background: To assess the efficacy profile of erlotinib-based doublet targeted therapy compared with erlotinib monotherapy for previously treated patients with advanced NSCLC, a meta-analysis was performed.

Patients and methods: We rigorously searched PubMed, Embase, Cochrane and meeting proceedings. Phase II/III randomized trials reporting on the efficacy of erlotinib-doublet therapy versus single-agent therapy were selected. We estimated the HR for OS, PFS and the RR for ORR, DCR, 1-year SR. Phases of trials, targeted signaling pathways, EGFR-status and KRAS- status were included in subset analysis.

Results: 24 studies involving 6,196 patients were eligible. In general, the combination targeted therapy significantly improved PFS, ORR and DCR. There was also a trend showing improved OS and 1-year SR in doublets group, though it was not statistically significant. Subgroup analysis suggested PFS improvement in EGFR wild-type, KRAS mutant, KRAS wild-type populations. Moreover, patients treated with anti-angiogenesis or anti-MET targeted agent revealed a significant benefit in PFS.

Conclusion: In patients with advanced NSCLC, erlotinib-doublets target therapy (specially combination with anti-angiogenesis and anti-MET targeted agents) was associated with a statistically significantly longer PFS, greater ORR and DCR, but the combination did not improve OS and 1-year SR compared with erlotinib alone.

\section{INTRODUCTION}

Based on the most recent WHO estimate, lung cancer is a leading cause of cancer-related mortality with approximately 1.59 million deaths worldwide in 2012. [1] In China, lung cancer is estimated to account for $21.6 \%$ of all cancer deaths in 2015. [2]

In patients with advanced non-small-cell lung cancer (NSCLC), platinum-doublet chemotherapy is standard treatment in the first-line setting; however, most patients ultimately progress and survived for less than 1 year. [3] Discovery and subsequent targeting of the epidermal growth factor receptor (EGFR) pathway has imparted clinical benefit and ushered in a new era of targeted therapeutic agents for patients with NSCLC. Several guidelines recommend EGFR tyrosine kinase inhibitors (TKIs), such as erlotinib, as an option of second- or thirdline treatments for advanced NSCLC, independent of the EGFR mutational status. [4] Nonetheless, prognosis remains poor; the median progression-free survival (PFS) for patients treated with erlotinib monotherapy, regardless of EFGR mutation status, is still only around 2.2 months after failure with platinum salts and overall survival was 6.7 months according to a placebo-controlled trial conducted by Shepherd et al. [5]

Multiple signaling pathways recognized to play key roles in homeostatic processes have been identified as key drivers of oncogenesis through genetic and 
epigenetic aberrations, including ErbB receptor tyrosine kinases, anaplastic lymphoma kinase (ALK), insulin-like growth factor-1 receptor (IGF-1R), hepatocyte growth factor (HGF)-mesenchymal-epithelial transition factor (MET) axis, to name a few. [6] Given the heterogeneity of NSCLC and potential crosstalk between signaling pathways implicated in tumor growth, angiogenesis and metastasis, combining targeted agents could improve the efficacy over single-target agents, which could also be necessary to reverse resistance to EGFR inhibitor therapy. [6-8]

Several trials have been conducted to evaluate benefits of combining targeted agent with erlotinib compared with erlotinib alone, especially the agents targeting angiogenesis, MET, IGF-1R and ErbB3 signaling. However, the results from these trials were controversial and some were of small sample size. This meta-analysis intended to pool and analyze all relevant randomized phase II/III trials, which provided a more precise assessment of efficacy of erlotinib-doublet targeted therapy compared with monotherapy in subsequent lines after previously treated with standard chemotherapy. Predefined subgroup analysis was conducted to identify the potential appropriate patient population to benefit from such combined therapy.

\section{RESULTS}

\section{Literature search}

We identified 2,740 initial article candidates, and 24 articles involving 6,196 patients met the inclusion criteria after rigorously identification (Figure 1). 2,656 articles were excluded based on the title and abstract for the following reasons: duplicates, irrelevant data, reviews, case reports, animal studies. The rest 84 articles were retrieved for full-text review, from which 60 were removed: 34 phase I trials, 24 single-arm phase II trials, 1 focusing on first-line therapy, 1 involving in a runin period where patients received the study drug. The remaining 17 trials [9-25] with full-text and 7 additional conference abstracts [26-32] were included in the final analysis.

\section{Study characteristics}

The detailed characteristics of eligible studies are summarized in Table 1 and Table 2. Of the 24 randomized trails, the primary end point was PFS in twelve [11,16$18,20,23,25,26,28,30-32]$, OS in six [12, 14, 21, 22, 24, 29], ORR in two [9, 10, 13, 27], ORR plus PFS (coprimary end points) in one [10], 12-weeks PFS rate in one[13], 4-momth PFS rate in one[15] and DCR at 3 months in one [19]. Six [12, 14, 21, 22, 24, 29] of the included studies were phase III RCTs and the remaining were phase II RCTs. 14 trials [10-15, 17, 18, 22-24, 26, 29 , 30] employed erlotinib plus placebo as the control arm, while the remaining 10 treated control subjects with single-agent erlotinib. 8 studies tested targeted therapies in molecularly enriched populations in accordance with EGFR status (immunocytochemistry positive [16]; wild-type [24, 31, 32]), KRAS status (wild-type) [25], expression of MET (immunocytochemistry 2+/3+)[29] and histological type (non-adenocarcinoma[21]; nonsquamous cell carcinoma [22, 24, 32]). Due to two threearm trials, each of which consisted of two comparisons with a shared control, there were four comparisons for OS and PFS from these two studies.[13,30] One article investigated two parallel randomized phase II trials, yet only one trial was of interest in our review.[25] All of the included studies provided sufficient data about OS, PFS and ORR except two[25, 28] without value of HR or $95 \%$ CI for survival data and one [30] without ORR. Data for DCR and 1-year SR were available in 16 [9-14, 16, 19-25, $31,32]$ and 17 [9-18, 21-24, 29, 31, 32] trials, respectively.

\section{Risk of bias}

All the included trials reported "randomization" with $75 \%$ and $54 \%$ studies providing the conduction details of random sequence generation and allocation concealment, respectively. $10 \mathrm{RCTs}$ were marked with "open-label" and the performance bias was assessed as "high risk". For other key domains, no high risk of bias was detected. Full details of the assessment are in Supplementary Table 1.

\section{Efficacy outcomes}

The median OS were 5.7 to 13.3 months in the combination arm versus 4.1 to 14 months in the control arm. Pooled HR for OS estimated from 22 studies was 0.96 (95\% CI 0.91-1.03, $p=0.26$; Figure 2). No significant heterogeneity was detected among the studies included for OS analysis $\left(I^{2}=31 \%\right)$.

The median PFS of the doublets group and singleagent group were 1.3 to 5.4 months and 1.5 to 3.5 months, respectively. Considering significant heterogeneity among the studies $\left(I^{2}=58 \%\right)$, a random effect model was employed to estimate the pooled HR for PFS. Pooled PFS of patients treated with erlotinib plus the other targeted agent was superior to those treated with erlotinib alone (HR 0.83, 95\% CI 0.75-0.91, $p=0.0002$; Figure 3).

1-year SR did not significantly improve with doublets compared with single erlotinib (RR 1.04, 95\% CI $0.97-1.12, p=0.27 ; I^{2}=25 \%$; Figure 4). However, ORR and DCR were in favor of the doublet targeted therapy (RR 1.28, $95 \%$ CI 1.08-1.52, $p=0.004 ; I^{2}=0 \%$; 
and RR 1.21, 95\% CI 1.13-1.30, $p<0.00001 ; I^{2}=44 \%$, respectively; Figures 5 and 6).

Neither phase II nor phase III trials subset analysis of OS revealed significant differences between the erlotinib-based combinations compared with the single agent (HR 0.91, $95 \%$ CI 0.82-1.01, $p=0.08 ; I^{2}=34 \%$; and HR 1.00, 95\% CI 0.92-1.08, $p=0.92 ; I^{2}=16 \%$, respectively; Table 3 ), whereas both phase II and phase III trials subgroup analysis showed improvement in PFS with doublets regimen over single erlotinb regimen (HR 0.83, $95 \%$ CI 0.73-0.95, $p=0.007 ; I^{2}=45 \%$; and HR 0.81, 95 $\%$ CI 0.69-0.96, $p=0.01 ; I^{2}=79 \%$, respectively; Table 3).

Various targeted signaling pathways were involved in the 24 eligible studies. For a subgroup analysis, we divided different targets into six groups: anti-angiogenesis, anti-MET, anti-IGF-1R, anti-ErbB3 signaling, anti- angiogenesis plus anti-MET signaling and others. Overall, no significant differences existed in PFS or OS between combining targeted therapy and erlotinib monotherapy, except that patients treated with erlotinib plus antiangiogenesis or anti-MET targeted agents showed improvement in PFS (HR 0.73, 95\% CI 0.62-0.86, $p=$ $0.0002 ; I^{2}=49 \%$; and HR 0.84, 95\% CI: 0.72-0.99, $p=$ $0.03 ; I^{2}=54 \%$, respectively) and the doulets erlotinib plus cabozantinib (anti-angiogenesis plus anti-MET signaling) group revealed significant improvement in both OS and PFS (HR 0.44, $95 \%$ CI 0.29-0.66, $p<0.0001$; and HR 0.35 , $95 \%$ CI 0.24-0.52, $p<0.00001$, respectively; Supplementary Figures 1 and 2; Table 3).

11 studies provided the detailed analysis of OS in EGFR wild-type population. The pooled HR was 0.89 (95\% CI $0.75-1.06, p=0.2 ; I^{2}=61 \%$; Supplementary

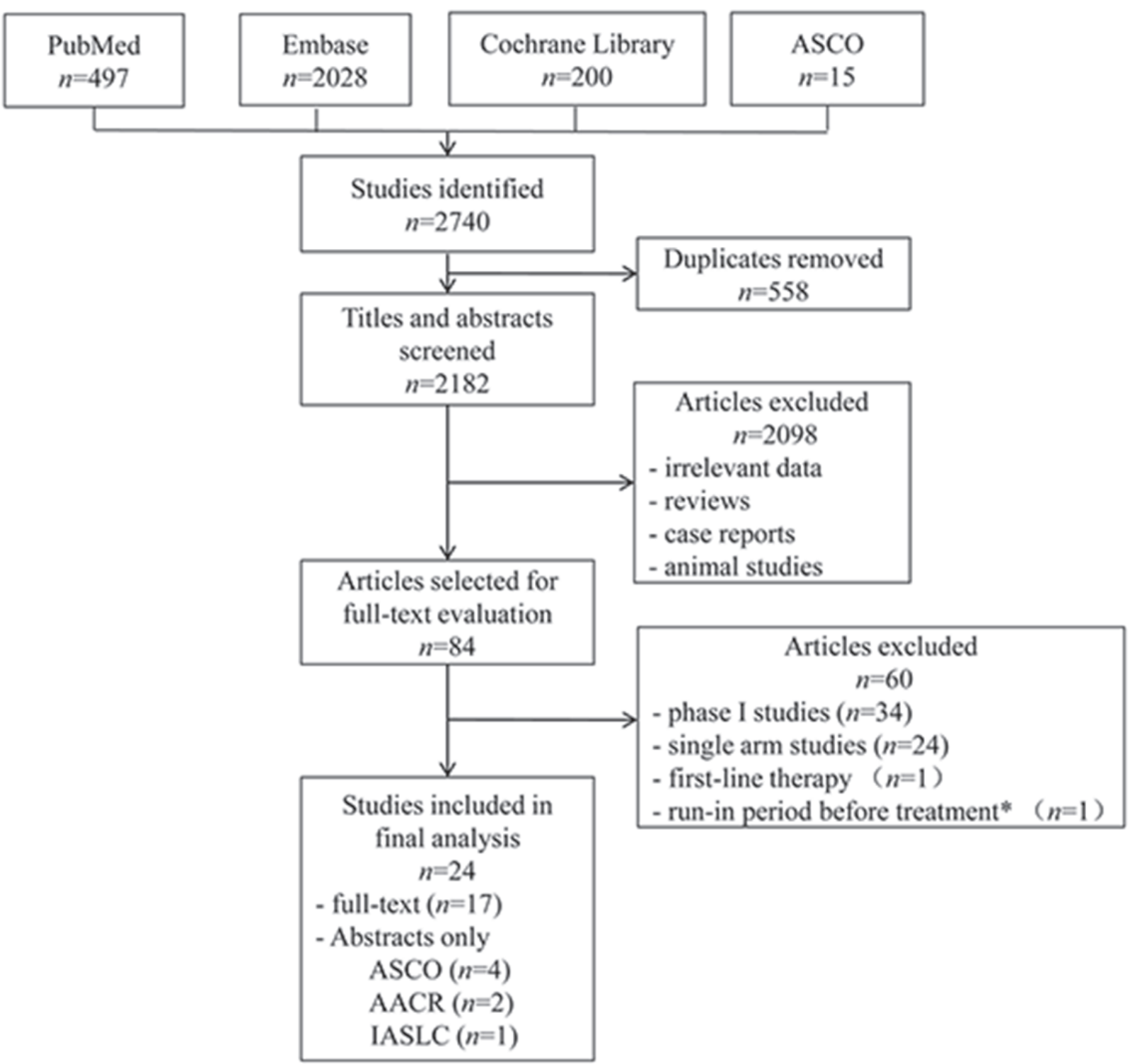

Figure 1: Flowchart of the process for selecting relevant articles. ASCO, American Society of Clinical Oncology; AACR, American Association for Cancer Research; IASLC, International Association for the Study of Lung Cancer. *Patients entered an openlabel run-in period where they received single-agent apricoxib $(400 \mathrm{mg} /$ day $)$ for 5 consecutive days. 
Table 1: Study characteristics of the randomized trials Included in the meta-analysis

\begin{tabular}{|c|c|c|c|c|c|c|c|c|c|c|c|c|c|}
\hline Study & Year & Phase & Group & $\begin{array}{l}\text { Targeted } \\
\text { signaling }\end{array}$ & \begin{tabular}{|l} 
Selected \\
populations
\end{tabular} & $\mathbf{N}$ & $\begin{array}{c}\text { Age, } \\
\text { years }\end{array}$ & $\begin{array}{l}\text { Female, } \\
\%\end{array}$ & $\begin{array}{l}\text { Smoking, } \\
\%\end{array}$ & $\begin{array}{l}\text { Histology, } \\
\text { AC/SCC, } \\
\%\end{array}$ & $\begin{array}{l}\text { ECOG } \\
\text { PS,0/1,\% }\end{array}$ & $\begin{array}{l}\text { Stage, IIIB/ } \\
\text { IV, \% }\end{array}$ & $\begin{array}{l}\text { prior } \\
\text { chemotherapy } \\
\text { regimens, } \\
1 / \geq 2, \%\end{array}$ \\
\hline \multirow{2}{*}{ Lynch[9] } & 2009 & II & Erl + bortezomib & \multirow{2}{*}{$\begin{array}{l}\text { proteasome } \\
\text { inhibitor }\end{array}$} & \multirow{2}{*}{ unselected } & 25 & 62 & 56 & 84 & $60 / 28$ & $29 / 67$ & $16 / 84$ & $4(0) / 76 / 20$ \\
\hline & & & Erl & & & 25 & 64 & 48 & 80 & $56 / 28$ & $28 / 72$ & $12 / 88$ & $12(0) / 84 / 4$ \\
\hline \multirow{2}{*}{ Herbst[12] } & 2011 & III & Erl + bevacizumab & \multirow{2}{*}{$\begin{array}{l}\text { anti-VEGF } \\
\text { monoclonal } \\
\text { antibody }\end{array}$} & \multirow{2}{*}{ unselected } & 319 & 65 & 46 & 89 & $76 / 3$ & $41 / 52$ & NA & NA \\
\hline & & & Erl + placebo & & & 317 & 65 & 46 & 90 & $74 / 5$ & $38 / 56$ & NA & NA \\
\hline \multirow{3}{*}{ Ramalingam[13] } & 2011 & II & $\begin{array}{l}\text { Erl + R1507 (9 mg/ } \\
\mathrm{kg} / \mathrm{wk})\end{array}$ & \multirow{3}{*}{$\begin{array}{l}\text { anti-IGF-1R } \\
\text { monoclonal } \\
\text { antibody }\end{array}$} & \multirow{3}{*}{ unselected } & 57 & 63 & 32 & 86 & $46 / 26$ & NA & $19 / 81$ & $77 / 23$ \\
\hline & & & $\begin{array}{l}\mathrm{Erl}+\mathrm{R} 1507(16 \mathrm{mg} / \\
\mathrm{kg} / 3 \mathrm{wks})\end{array}$ & & & 57 & 62 & 33 & 91 & $44 / 28$ & NA & $12 / 88$ & $68 / 32$ \\
\hline & & & $\mathrm{Erl}+$ placebo & & & 57 & 62 & 35 & 84 & $63 / 21$ & NA & $19 / 81$ & $75 / 25$ \\
\hline \multirow{2}{*}{ Sequist[11] } & 2011 & II & Erl + tivantinib & \multirow{2}{*}{ MET inhibitor } & \multirow{2}{*}{ unselected } & 84 & 64 & 39 & 80 & $56 / 31$ & $27 / 71$ & $10 / 91$ & $60 / 40$ \\
\hline & & & Erl + placebo & & & 83 & 62 & 41 & 78 & $65 / 29$ & $20 / 80$ & $13 / 87$ & $61 / 39$ \\
\hline \multirow{2}{*}{ Spigel[10] } & 2011 & II & Erl + sorafenib & \multirow{2}{*}{$\begin{array}{l}\text { TKI against } \\
\text { VEGFR2/3, } \\
\text { PDGFRB }\end{array}$} & \multirow{2}{*}{ unselected } & 111 & 65 & 44 & 83 & $\mathrm{NA} / 33$ & $29 / 56$ & NA & $66 / 34$ \\
\hline & & & Erl + placebo & & & 55 & 65 & 53 & 85 & $\mathrm{NA} / 31$ & $29 / 51$ & NA & $51 / 49$ \\
\hline \multirow{2}{*}{ Scagliotti[14] } & 2012 & III & Erl + sunitinib & \multirow{2}{*}{$\begin{array}{l}\text { TKI against } \\
\text { VEGFR, } \\
\text { PDGFRA/B }\end{array}$} & uncelected & 480 & 61 & 38 & 80 & $57 / 28$ & $38 / 61$ & 9/91 & $71 / 29$ \\
\hline & & & Erl + placebo & & & 480 & 61 & 41 & 81 & $54 / 28$ & $37 / 63$ & $7 / 93$ & $71 / 29$ \\
\hline Spigel/IASLC[26] & 2012 & II & Erl + pazopanib & $\begin{array}{l}\text { TKI against } \\
\text { VEGFR. }\end{array}$ & unselected & 134 & 66 & 47 & 96 & $\mathrm{NA} / 22$ & NA & NA & $61 / 39$ \\
\hline & & & Erl + placebo & | PDGFRA/B & & 67 & 67 & 42 & 91 & $\mathrm{NA} / 26$ & NA & NA & $65 / 35$ \\
\hline Witta[15] & 2012 & II & Erl + entinostat & HDACi & unselected & 67 & 66 & 42 & 84 & $58 / 27$ & $43 / 45$ & NA & NA \\
\hline Witta[1J] & & & Erl + placebo & | ПDAC1 & unserected & 65 & 67 & 34 & 83 & $43 / 32$ & $34 / 52$ & NA & NA \\
\hline Relani[16] & 2013 & II & Erl + PF-3512676 & TI RO agonist & EGFR-IHC & 21 & 63 & 57 & 90 & $62 / 33$ & $90(0 / 1)$ & NA & $57 / 43$ \\
\hline Belanı[16] & & & Erl & TLRY agonist & positive & 22 & 64 & 41 & 86 & $64 / 9$ & $91(0 / 1)$ & NA & $86 / 14$ \\
\hline ירברז & 2013 & II & $\mathrm{Erl}+$ fulvestrant & Estrogen & L & 72 & NA & NA & NA & NA & NA & NA & NA \\
\hline Garon/AACR[27] & & & Erl & antagonist & unselected & 34 & $\overline{\mathrm{NA}}$ & NA & NA & NA & NA & NA & NA \\
\hline & 2013 & II & Erl + sunitinib & TKI against & & 65 & 59 & 40 & 88 & $55 / 23$ & $32 / 66$ & $2 / 97$ & $60 / 37$ \\
\hline Groen[18] & & & Erl + placebo & $\begin{array}{l}\text { VEGFR, } \\
\text { PDGFRA/B }\end{array}$ & unserected & 67 & 61 & 33 & 85 & $46 / 28$ & $31 / 67$ & $0 / 100$ & $69 / 31$ \\
\hline Sniol[177] & 2013 & II & Erl + onartuzumab & anti-MET & L & 69 & 64 & 42 & 86 & $58 / 29$ & $32 / 62$ & NA & NA \\
\hline Spigel[1/] & & & Erl + placebo & $\begin{array}{l}\text { monocional } \\
\text { antibody }\end{array}$ & unselected & 68 & 63 & 38 & 88 & $61 / 29$ & $31 / 66$ & NA & NA \\
\hline Do $5[107$ & 2014 & II & Erl + everolimus & TOP i & L & 66 & 60 & 46 & 80 & $70 / 15$ & NA & $12 / 78$ & $77 / 23$ \\
\hline Besse[19] & & & Erl & mIOR inhibitor & unselected & 67 & 61 & 50 & 81 & $69 / 15$ & NA & $19 / 63$ & $61 / 37$ \\
\hline & 2014 & II & Erl + dalotuzumab & anti-IGF-1R & & 37 & 62 & 27 & 89 & $38 / 30$ & $30 / 65$ & $11 / 89$ & NA \\
\hline Moran[20] & & & Erl & $\begin{array}{l}\text { monoclonal } \\
\text { antibody }\end{array}$ & unselected & 38 & 59 & 26 & 71 & $40 / 16$ & $34 / 63$ & $24 / 76$ & NA \\
\hline 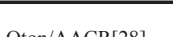 & 2014 & II & Erl + Efatutazone & PDAP & Luncelocted & 45 & 60 & 24 & 69 & NA & NA & NA & NA \\
\hline Oton/AACR $[28]$ & & & Erl & PPAR $\gamma$ agonist & unselected & 45 & 61 & 44 & 54 & NA & NA & NA & NA \\
\hline & 2014 & II & $\begin{array}{l}\text { Erl + patritumab (18 } \\
\mathrm{mg} / \mathrm{kg} / 3 \text { wks })\end{array}$ & & & 70 & 62 & 46 & 86 & $66 / 27$ & $47 / 53$ & NA & $71 / 29$ \\
\hline Pawel/ASCO[30] & & & $\begin{array}{l}\text { Erl + patritumab }(9 \mathrm{mg} / \\
\mathrm{kg} / 3 \mathrm{wks})\end{array}$ & $\begin{array}{l}\text { monoclonal } \\
\text { antibody }\end{array}$ & unselected & 71 & 65 & 32 & 85 & $62 / 32$ & $42 / 58$ & NA & $68 / 32$ \\
\hline & & & Erl + placebo & & & 71 & 60 & 39 & 93 & $60 / 30$ & $35 / 65$ & NA & $66 / 34$ \\
\hline S- & 2014 & II & Erl + MM-121 & anti-ErbB3 & LUT & 85 & 65 & 41 & 84 & NA & NA & NA & $32 / 68$ \\
\hline Sequist/Asco[31] & & & Erl & $\begin{array}{l}\text { monocional } \\
\text { antibody }\end{array}$ & WI-EUFK & 44 & 64 & 39 & 71 & NA & NA & NA & $39 / 61$ \\
\hline Snicel/ASCOГ29] & 2014 & III & Erl + onartuzumab & anti-MET & MET-IHC & 250 & 62 & 44 & NA & $\mathrm{NA} / 16$ & $37 / 61$ & NA & NA \\
\hline Splgel/ASCO[29] & & & Erl + placebo & antibody & $2+/ 3$ & 249 & 63 & 44 & NA & $\mathrm{NA} / 12$ & $31 / 68$ & NA & NA \\
\hline $\mathrm{N}$ & 2015 & II & Erl + cabozantinib & TKI against & non-SCC, & 36 & 63 & NA & 83 & NA & $25 / 64$ & NA & NA \\
\hline Neal/ASC U [32] & & & Erl & MET,VEĞFR2 & WT-EGFR & 38 & 66 & NA & 87 & NA & $24 / 63$ & NA & NA \\
\hline Peckamp[23] & 2015 & II & Erl + celecoxib & COX-2 & unselected & 54 & 64 & 52 & 63 & $59 / 11$ & $48 / 52$ & $11 / 89$ & $11(0) / 50 / 39$ \\
\hline Кескаптр[23] & & & Erl + placebo & inhibitor & unserected & 53 & 65 & 55 & 62 & $60 / 9$ & $49 / 51$ & $8 / 92$ & $13(0) / 51 / 36$ \\
\hline Scagliotti-fig[21] & 2015 & III & Erl + figitumumab & $\begin{array}{l}\text { anti-IGF-1R } \\
\text { monoclonal }\end{array}$ & non-AC & 293 & 62 & 22 & 94 & $0 / 90$ & $81(0 / 1)$ & $21 / 78$ & NA \\
\hline & & & Erl & antibody & & 290 & 62 & 22 & 91 & $0 / 91$ & $82(0 / 1)$ & $19 / 81$ & NA \\
\hline Scogliottitiv[201 & 2015 & III & Erl + tivantinib & MFT inbibitor & non_SCC & 526 & 62 & 41 & 81 & $91 / 0$ & $32 / 68$ & $4 / 95$ & $66 / 34$ \\
\hline Sedagioull-tiv [22] & & & $\mathrm{Erl}+$ placebo & IVIE I IIIIIOItor & HOHI-SCL & 522 & 61 & 41 & 81 & $95 / 0$ & $32 / 68$ & $3 / 96$ & $67 / 33$ \\
\hline Yochiok [24] & 2015 & III & Erl + tivantinib & MET inhibitor & $\mathrm{CC}$ & 154 & 63 & 29 & 73 & NA & $43 / 57$ & $4 / 96$ & $60 / 40$ \\
\hline 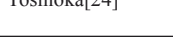 & & & Erl + placebo & LIE TIIIUTIOU & WT- & 153 & 63 & 33 & 75 & NA & $33 / 67$ & $6 / 94$ & $59 / 41$ \\
\hline Carter[25] & 2016 & II & Erl + selumetinib & MEK kinase & WTTKRAS & 19 & 84 & 47 & 64 & $79 / 21$ & $10 / 37$ & NA & $42 / 58$ \\
\hline Carter[25] & & & Erl & inhibitor & WI-KRAS & 19 & 68 & 32 & 64 & $79 / 21$ & $10 / 58$ & NA & $52 / 48$ \\
\hline
\end{tabular}

ECOG PS, Eastern Cooperative Oncology Group Performance Status; Erl, erlotinib; VEGF, vascular endothelial growth factor; VEGFR, vascular endothelial growth factor receptor; IGF-1R, insulin-like growth factor-1 receptor; HDACi, selective histone deacetylase inhibitor; MET, mesenchymal-epithelial transition factor; TKI, tyrosine kinase inhibitor; PDGFR, platelet-derived growth factor receptor; TLR9, Toll-like receptor 9; mTOR, mammalian target of rapamycin; PPAR $\gamma$, peroxisome proliferative activated receptor $\gamma$; COX-2, cyclo-oxygen-ase-2; MEK, AC, adenocarcinoma; SCC, squamous cell carcinoma;

EGFR, epidermal growth factor receptor; IHC, immunohistochemistry; WT, wild-type; NA, not applicable; 
Table 2: Study outcomes of the randomized trials included in the meta-analysis

\begin{tabular}{|c|c|c|c|c|c|c|c|c|c|c|c|c|c|}
\hline \multirow{2}{*}{ Study } & \multirow{2}{*}{ Group } & \multirow{2}{*}{ Primary endpoint } & \multirow{2}{*}{ ORR, \% } & \multirow{2}{*}{ DCR, \% } & \multirow{2}{*}{$\begin{array}{c}1 \text {-year } \\
\text { SR, } \%\end{array}$} & \multirow{2}{*}{$\begin{array}{c}\text { OS, } \\
\text { mo }\end{array}$} & \multirow{2}{*}{$\begin{array}{r}\text { PFS, } \\
\text { mo }\end{array}$} & \multicolumn{3}{|c|}{ WT-EGFR } & \multicolumn{3}{|c|}{ Mut-EGFR } \\
\hline & & & & & & & & $\mathrm{N}$ & OS, mo & PFS, mo & $\mathbf{N}$ & OS, mo & PFS, mo \\
\hline \multirow{2}{*}{ Lynch[9] } & Erl + bortezomib & ORR & 8.0 & 40.0 & 30 & 8.5 & 1.3 & 12 & NA & NA & 2 & NA & NA \\
\hline & Erl & & 16.0 & 52.0 & 40 & 7.3 & 2.7 & 11 & NA & NA & 4 & NA & NA \\
\hline \multirow{2}{*}{ Herbst[12] } & Erl + bevacizumab & OS & 11.9 & 42.6 & 42.1 & 9.3 & 3.4 & 173 & 8.1 & NA & 12 & NA & NA \\
\hline & Erl + placebo & & 6.0 & 32.8 & 40.7 & 9.2 & 1.7 & 152 & 9.1 & NA & 18 & 20.2 & NA \\
\hline \multirow{3}{*}{ Ramalingam[13] } & $\mathrm{Erl}+\mathrm{R} 1507(9 \mathrm{mg} / \mathrm{kg} / \mathrm{wk})$ & \multirow{3}{*}{ 12-wk PFS rate } & 8.8 & 49.1 & 30.1 & 8.1 & 1.9 & $\mathrm{NA}$ & NA & NA & 2 & NA & NA \\
\hline & $\mathrm{Erl}+\mathrm{R} 1507(16 \mathrm{mg} / \mathrm{kg} / 3 \mathrm{wks})$ & & 7.0 & 56.1 & 50.6 & 12.1 & 2.7 & $\mathrm{NA}$ & NA & NA & 1 & NA & NA \\
\hline & Erl + placebo & & 8.8 & 49.1 & 33.1 & 8.1 & 1.5 & $\mathrm{NA}$ & $\mathrm{NA}$ & NA & 3 & NA & $\mathrm{NA}$ \\
\hline \multirow{2}{*}{ Sequist[11] } & Erl + tivantinib & PFS & 8.3 & 57.1 & 28.3 & 8.5 & 3.8 & 51 & NA & 3.2 & 6 & NA & 5.6 \\
\hline & Erl + placebo & & 6.0 & 47.0 & 32.4 & 6.9 & 2.3 & 48 & NA & 1.9 & 11 & NA & 4.9 \\
\hline \multirow{2}{*}{ Spigel[26] } & Erl + sorafenib & Oopripect & 8.1 & 54.1 & 32.7 & 7.6 & 3.4 & 43 & 8.1 & 3.4 & 2 & NA & NA \\
\hline & Erl + placebo & ORK/PFS & 10.9 & 38.2 & 40.3 & 7.2 & 1.9 & 24 & 4.5 & 1.8 & 3 & NA & 9.2 \\
\hline Soulioni141 & Erl + sunitinib & OS & 10.6 & 42.9 & 40 & 9.0 & 3.6 & $\mathrm{NA}$ & NA & NA & NA & NA & NA \\
\hline Scagiottri[14] & Erl + placebo & & 6.9 & 35.0 & 37 & 8.5 & 2.0 & $\mathrm{NA}$ & NA & NA & NA & NA & NA \\
\hline Sniol/L SI CDO & Erl + pazopanib & PFS & 9.0 & NA & NA & 6.8 & 2.6 & $\mathrm{NA}$ & NA & NA & NA & NA & NA \\
\hline Spigel//ASLC [26] & Erl + placebo & & 4.5 & NA & NA & 6.7 & 1.8 & $\mathrm{NA}$ & NA & NA & NA & NA & NA \\
\hline Witur $[15]$ & Erl + entinostat & $D_{1}$ & 3.0 & NA & 39.2 & 8.9 & 2.0 & 33 & NA & NA & 3 & NA & NA \\
\hline Witta $[1 T]$ & Erl + placebo & 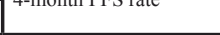 & 9.2 & NA & 28.9 & 6.7 & 1.9 & 43 & NA & NA & 3 & NA & NA \\
\hline Dabirig & Erl + PF-3512676 & PFS & 9.5 & 19.1 & 34.3 & 6.4 & 1.6 & 9 & NA & NA & 4 & NA & 1.6 \\
\hline Belam[10] & Erl & & 4.6 & 18.2 & 15.3 & 4.7 & 1.7 & 14 & NA & NA & 2 & NA & 1.7 \\
\hline 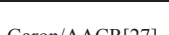 & Erl + fulvestrant & ORR & 23.6 & $\mathrm{NA}$ & NA & 9.4 & 1.9 & 38 & 7.4 & 2.0 & 14 & NA & NA \\
\hline Jaron/AAC K[2'] & Erl & & 14.7 & NA & NA & 5.7 & 1.8 & 14 & 5.9 & 1.6 & 7 & NA & NA \\
\hline Conon[18] & Erl + sunitinib & PFS & 4.6 & $\mathrm{NA}$ & 32 & 8.2 & 2.8 & 21 & $\mathrm{NA}$ & NA & 4 & NA & $\mathrm{NA}$ \\
\hline Groen[18] & Erl + placebo & & 3.0 & $\mathrm{NA}$ & 42 & 7.6 & 2.0 & 19 & NA & NA & 1 & NA & NA \\
\hline Snimlivit & Erl + onartuzumab & PFS & 5.8 & $\mathrm{NA}$ & 36 & 8.9 & 2.2 & 49 & 8.5 & NA & 10 & NA & NA \\
\hline Spigen[1]] & Erl + placebo & & 4.4 & $\mathrm{NA}$ & 30.7 & 7.4 & 2.6 & 50 & 7.4 & NA & 9 & NA & NA \\
\hline 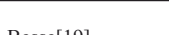 & Erl + everolimus & $D_{C P D}-2$ & 12.1 & 57.6 & NA & 9.1 & 2.9 & $\mathrm{NA}$ & NA & NA & NA & NA & NA \\
\hline Besse[19] & Erl & DCK at $J$ montis & 10.5 & 38.8 & NA & 9.7 & 2.0 & $\mathrm{NA}$ & NA & NA & NA & NA & NA \\
\hline Moren[20] & Erl + dalotuzumab & PFS & 2.7 & 59.5 & NA & 6.6 & 2.5 & $\mathrm{NA}$ & NA & NA & NA & NA & NA \\
\hline Moran[ 20$]$ & Erl & & 7.9 & 63.2 & NA & 10.2 & 1.6 & $\mathrm{NA}$ & NA & NA & NA & NA & NA \\
\hline 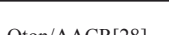 & Erl + Efatutazone & PFS & 20.5 & NA & NA & 7.6 & 4.1 & $\mathrm{NA}$ & NA & NA & NA & NA & NA \\
\hline Iton/AACR $[28]$ & Erl & & 20.0 & NA & NA & 11.4 & 2.8 & $\mathrm{NA}$ & NA & $\mathrm{NA}$ & NA & NA & NA \\
\hline & Erl + patritumab $(18 \mathrm{mg} / \mathrm{kg} / 3 \mathrm{wks})$ & PFS & NA & NA & NA & NA & 1.4 & 17 & NA & NA & 0 & NA & NA \\
\hline Pawel/ASCO[30] & Erl + patritumab $(9 \mathrm{mg} / \mathrm{kg} / 3 \mathrm{wks})$ & & NA & NA & NA & NA & 2.5 & 21 & NA & NA & 2 & NA & NA \\
\hline & Erl + placebo & & NA & $\mathrm{NA}$ & NA & NA & 1.6 & 23 & NA & NA & 2 & NA & NA \\
\hline S c. & Erl + MM-121 & PFS & 4.7 & 40.0 & 27.1 & 6.3 & 1.9 & 85 & 6.3 & 1.9 & 0 & NA & NA \\
\hline 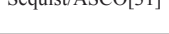 & Erl & & 4.6 & 29.6 & 24.8 & 9.3 & 1.8 & 44 & 9.3 & 1.8 & 0 & NA & NA \\
\hline $\mathrm{Ser}_{\mathrm{i}}$ & Erl + onartuzumab & OS & 8.4 & $\mathrm{NA}$ & 27.3 & 6.8 & 2.7 & 222 & 6.4 & 2.6 & 28 & 12.6 & NA \\
\hline Spreel/AsC $[29]$ & Erl + placebo & & 9.6 & $\mathrm{NA}$ & 33.0 & 9.1 & 2.6 & 220 & 7.8 & 1.5 & 29 & NA & 8.5 \\
\hline דרכו & Erl + cabozantinib & PFS & 5.6 & 36.1 & 58.8 & 13.3 & 4.7 & 36 & 13.3 & 4.7 & 0 & NA & NA \\
\hline Neal/ASCO [32] & Erl & & 2.6 & 15.8 & 17.6 & 4.1 & 1.9 & 38 & 4.1 & 1.9 & 0 & NA & NA \\
\hline Perkomn[23] & Erl + celecoxib & PFS & 22.2 & 63.0 & 53.7 & 12.9 & 5.4 & 31 & 9.8 & 3.2 & 12 & NA & 9.2 \\
\hline Keckamp[23] & Erl + placebo & & 32.1 & 56.6 & 60.4 & 14 & 3.5 & 27 & 10.9 & 1.8 & 14 & NA & 9.2 \\
\hline Sealiontifo[21] & Erl + figitumumab & OS & 5.5 & 44.0 & 24.5 & 5.7 & 2.1 & $\mathrm{NA}$ & NA & NA & NA & NA & NA \\
\hline Scagiotil-ng[21] & Erl & & 3.8 & 48.6 & 24.9 & 6.2 & 2.6 & $\mathrm{NA}$ & NA & NA & $\mathrm{NA}$ & NA & NA \\
\hline Sorlisti. & Erl + tivantinib & OS & 10.3 & 45.8 & 35.9 & 8.5 & 3.6 & 469 & 7.2 & 2.7 & 56 & NA & NA \\
\hline Scagiotti-tiv[22] & Erl+ placebo & & 6.5 & 32.0 & 34.1 & 7.8 & 1.9 & 468 & 7.1 & 1.9 & 53 & $\mathrm{NA}$ & NA \\
\hline Y & Erl + tivantinib & OS & 8.4 & 39.0 & 54.4 & 12.7 & 2.9 & 154 & 12.7 & 2.9 & 0 & NA & NA \\
\hline Yosnioka[24] & Erl + placebo & & 6.5 & 32.0 & 47.6 & 11.1 & 2.0 & 153 & 11.1 & 2.0 & 0 & NA & NA \\
\hline$C_{0}=-5=0$ & Erl + selumetinib & PFS & 12.0 & 35.0 & NA & 12.9 & 2.1 & 18 & NA & NA & 1 & NA & NA \\
\hline Carter[23] & Erl & & 5.0 & 47.0 & NA & 6.3 & 2.4 & 18 & NA & NA & 1 & NA & NA \\
\hline
\end{tabular}

ORR, objective response rate; DCR, disease control rate; SR, survival rate; OS, overall survival; PFS, progression-free survival; WT, wild-type; Mut, mutant; mo, months; Erl, erlotinib; wk, weeks; NA, not applicable; EGFR, epidermal growth factor receptor; ASCO, American Society of Clinical Oncology; AACR, American Association for Cancer Research; IASLC, International Association for the Study of Lung Cancer

Figure 3). Combining PFS of ten trials involving 2205 NSCLC harboring wild-type EGFR produced a significant improvement from the doublet targeted therapy (HR 0.68,
95\% CI 0.57-0.83, $p<0.0001 ; I^{2}=64 \%$; Supplementary Figure 4). Complete survival results of subgroup analysis based on EGFR gene mutations, protein expression and 
Table 3: Sub-group analysis based on study characteristics.

\begin{tabular}{|c|c|c|c|c|c|c|c|c|}
\hline \multirow[b]{2}{*}{ Sub-group } & \multicolumn{4}{|c|}{ OS } & \multicolumn{4}{|c|}{ PFS } \\
\hline & $N$ & HR $(95 \% C I)$ & $p$ & $\underset{\%}{\text { I-square, }}$ & $N$ & HR $(95 \% C I)$ & $p$ & $\begin{array}{c}\text { I-square, } \\
\%\end{array}$ \\
\hline \multicolumn{9}{|l|}{ Phase } \\
\hline II & 2035 & $0.91(0.82,1.01)$ & 0.08 & 34 & 2035 & $0.83(0.73,0.95)$ & 0.007 & 45 \\
\hline III & 4033 & $1.00(0.92,1.08)$ & 0.92 & 16 & 4033 & $0.81(0.69,0.96)$ & 0.01 & 79 \\
\hline model & \multicolumn{4}{|c|}{ IV, fixed-effects model } & \multicolumn{4}{|c|}{ IV, random-effects model } \\
\hline \multicolumn{9}{|l|}{ Mechanism } \\
\hline Anti-angiogenesis & 2095 & $0.96(0.86,1.06)$ & 0.42 & 0 & 2095 & $0.73(0.62,0.86)$ & 0.0002 & 49 \\
\hline Anti-MET & 2158 & $0.99(0.86,1.13)$ & 0.86 & 24 & 2158 & $0.84(0.72,0.99)$ & 0.03 & 54 \\
\hline $\begin{array}{l}\text { Anti-angiogenesis \& } \\
\text { anti-MET }\end{array}$ & 74 & $0.44(0.29,0.66)$ & $<0.0001$ & NA* & 74 & $0.35(0.24,0.52)$ & $<0.00001$ & NA* \\
\hline Anti-IGF-1R & 829 & $0.98(0.73,1.30)$ & 0.88 & 57 & 829 & $1.04(0.90,1.21)$ & 0.55 & 0 \\
\hline Anti-ErbB3 & 341 & $1.12(0.89,1.43)$ & 0.34 & 0 & 341 & $0.85(0.68,1.06)$ & 0.16 & 0 \\
\hline Others & 571 & $0.91(0.74,1.13)$ & 0.4 & 0 & 571 & $0.91(0.96,1.09)$ & 0.31 & 0 \\
\hline model & \multicolumn{4}{|c|}{ IV, random-effects model } & \multicolumn{4}{|c|}{ IV, random-effects model } \\
\hline \multicolumn{9}{|l|}{$E G F R$ status } \\
\hline Mutant & 196 & $1.01(0.32,3.19)$ & 0.98 & 65 & 105 & $1.09(0.63,1.88)$ & 0.76 & 0 \\
\hline Wild-type & 2589 & $0.89(0.75,1.06)$ & 0.2 & 61 & 2205 & $0.68(0.57,0.83)$ & $<0.0001$ & 64 \\
\hline IHC-positive & 297 & $1.10(0.83,1.46)$ & 0.51 & 0 & 108 & $0.92(0.58,1.47)$ & 0.73 & 0 \\
\hline IHC-negative & 91 & $0.92(0.56,1.50)$ & 0.74 & NA* & 31 & $0.95(0.37,2.47)$ & 0.92 & $\mathrm{NA}^{*}$ \\
\hline FISH-positive & 105 & $1.34(0.85,2.12)$ & 0.21 & 0 & 36 & $0.90(0.41,1.97)$ & 0.79 & NA* \\
\hline FISH-negative & 158 & $0.90(0.47,1.71)$ & 0.74 & 52 & 102 & $0.87(0.54,1.41)$ & 0.58 & 0 \\
\hline model & \multicolumn{4}{|c|}{ IV, random-effects model } & \multicolumn{4}{|c|}{ IV, random-effects model } \\
\hline \multicolumn{9}{|l|}{ KRAS status } \\
\hline Mutant & 499 & $0.95(0.76,1.19)$ & 0.64 & 34 & 102 & $0.23(0.13,0.41)$ & $<0.00001$ & 0 \\
\hline Wild-type & 1530 & $0.93(0.82,1.05)$ & 0.23 & 0 & 523 & $0.79(0.64,0.97)$ & 0.03 & 12 \\
\hline model & \multicolumn{4}{|c|}{ IV, fixed-effects model } & \multicolumn{4}{|c|}{ IV, fixed-effects model } \\
\hline
\end{tabular}

OS, overall survival; PFS, progression-free survival; HR, hazard ratio; CI, confidence interval; IV, inverse variance; I-square, inconsistency statistic; MET, mesenchymal-epithelial transition factor; IGF-1R, insulin-like growth factor-1 receptor; EGFR, epidermal growth factor receptor; IHC, immunohistochemistry; FISH, fluorescence in-situ hybridization;

*NA, not applicable, due to only one trail involved

gene copy number were summarized in Table 3. No significant differences were observed expect for PFS in EGFR wild-type population mentioned above.

In patients with KRAS mutations, the pooled HR for OS and PFS for combination arm versus erlotinib arm were $0.95\left(95 \%\right.$ CI $\left.0.76-1.19, p=0.64 ; I^{2}=34 \%\right)$ and 0.23 (95\% CI 0.13-0.41, $p<0.00001 ; I^{2}=0 \%$ ), respectively. In $K R A S$ wild-type population, the pooled HR for OS and PFS were $0.93\left(95 \%\right.$ CI $\left.0.82-1.05, p=0.23 ; I^{2}=0 \%\right)$ and 0.79 (95\% CI $0.64-0.97, p=0.03 ; I^{2}=12 \%$ ), respectively (Supplementary Figures 5 and 6; Table 3).

\section{Publication bias}

After assessment by Begg's test and Egger's test, no publication bias was found. The $p$ values based on Begg's test for OS, PFS, ORR, DCR, 1-year SR in the total population were $0.941,0.309,0.712,0.449,0.387$, respectively. For Egger's test, the p values were 0.768 , $0.673,0.166,0.701,0.521$, respectively.

\section{DISCUSSION}

EGFR inhibitors have been approved for the second-line treatment of advanced NSCLC, regardless of EGFR mutational status.[4] However, patients who initially benefit from EGFR-targeted therapy eventually develop resistance and have poor prolongation of survival. Currently, there are multiple trails combining molecular agents that target different signaling pathways, attempting to overcome drug resistance and optimize utilization of single-agent erlotinib.

Our meta-analysis focused on erlotinib-based doublets as subsequent treatment after disease progression with chemotherapy. We confirmed that combination therapy resulted in prolonged progression-free survival (PFS), better overall response rate (ORR) and disease control rate (DCR) as compared to erlotinib monotherapy, though similarities in overall survival and one-year survival rate were observed. Perhaps these results were not surprising because PFS, ORR and DCR were all tumorbased assessment end points, while OS analysis could 
be confounded by multiple factors such as cross-over, subsequent therapies and long post-progression survival. A recent study investigating trail-level associations between PFS, ORR and OS may supporting our viewpoint, which demonstrated a strong association between ORR and PFS, but no association existed between ORR and OS or between PFS and OS.[33]

Pan et al. had performed a meta-analysis about similar subjects based on published data updated in November 2012, which concluded that erlotinib-based doublets regimen significantly improved ORR and DCR compared with single erlotinib, but 1-year SR was not significantly improved for doublets.[34] Though these results were consistent with ours, only five studies involving 2,100 patients were included in the metaanalysis, while our study included 24 RCTs involving 6,196 patients. Furthermore, besides dichotomous data (ORR, DCR, 1-year SR), our study pooled the HR of time-to-event data (OS, PFS) as well, taking into account both the event and the timing of the event, to evaluate the efficacy of doublets therapy.

Qi et al. also conducted a meta-analysis evaluating combined targeted agents versus single-agent erlotinib, updated in May 2012. [35] The author included eight studies involving 2,417 patients and the efficacy endpoints were OS (HR 0.90, 95\% CI 0.82-0.99, $p=0.024$ ), PFS (HR 0.83, 95\% CI 0.72-0.97, $p=0.018$ ) and ORR (OR $1.35,95 \% \mathrm{CI}: 1.01-1.80, p=0.04)$, all of which were in favor of the doublet targeted therapy according to the author's analysis. Whereas, our pooled data showed no statistical difference existed in OS between two arms. Possible explanation for this inconsistency was that another sixteen trails were incorporated and the number of participants was approximately 2.5 -fold in our metaanalysis; Besides, the discordance might be associated with a three-arm trail investigating combing R1507 (given weekly or every 3 weeks) with erlotinib.[13] The trail reported HR for survival data with $90 \%$ confidence interval (CI), which should be transformed to $95 \%$ CI for further meta-analysis. For example, the $90 \%$ CI of HR for OS in 'weekly' group were 0.58-1.21 as reported yet it should be transformed to $95 \%$ CI, namely $0.54-1.30$. Consequently, the revised pooled HR along with $95 \%$ CI for OS and PFS in the meta-analysis conducted by Qi et al. were 0.90 (95\% CI 0.82-1.00, $p=0.04)$ and 0.82 $(95 \%$ CI $0.71-0.95, p=0.010)$. The revised $p$ value $(0.04)$ for pooled OS data, though statistically significant, was apparently larger than the author reported (0.024).

Subgroup analysis conducted by Qi et al. based on phases of trials, EGFR-status and KRAS status showed

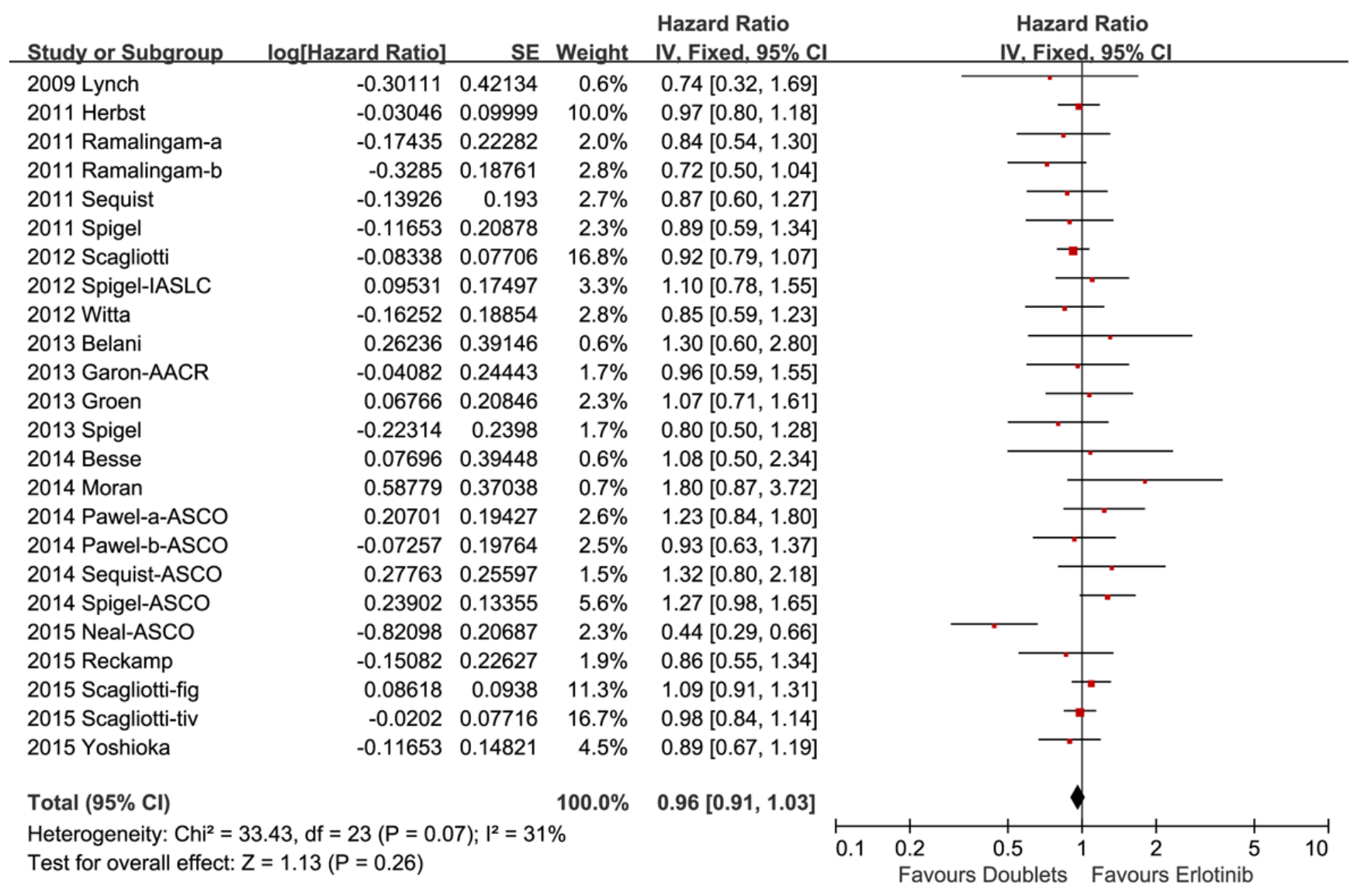

Figure 2: Forest plots for overall survival. 
that there was just a tendency to improve PFS and OS in doublets, except that PFS for patients with EGFRmutation or wild-type $K R A S$ favored single agent. All of these subset results were not statistically significant. However, given that mutational status was rarely reported according the included trails in Qi's article, results must be interpreted with caution. Conversely, we performed similar subset analysis based on a relatively large number of patients and strict definitions of EGFR status, that is gene mutant or wild-type, IHC positive or negative and fluorescence in-situ hybridization (FISH) positive or negative. Significantly, PFS improvement in doublets in EGFR wild-type $(p<0.0001), K R A S$ mutant $(p<$ $0.00001), K R A S$ wild-type ( $p=0.03$ ) was observed; While, PFS in EGFR-mutant patients showed a trend in favor of single-agent erlotinib (HR 1.09, 95\%CI 0.63-1.88). The mechanism underlying these observations were unclear.

MET, a transmembrane tyrosine kinase receptor, is central to the processes of cancer cell migration, invasion, proliferation, and metastasis.[36] MET amplification and/ or mutations are found in many human malignancies, including NSCLC, and predicts both resistane to EGFR TKIs and poor survival.[36-38] Thus, EGFR and MET may cooperate in driving tumorigenesis. Targeting angiogenesis is another promising strategy to improve survival in patients with many solid tumors, including NSCLC.[39]

Cabozantinib is a small molecule inhibitor of multiple receptor tyrosine kinases, including MET and vascular endothelial growth factor receptor 2 (VEGFR2). Notably, encouraging results of a randomized phase II trial testing cabozantinib, erlotinib or the combination in patients with EGFR wild-type NSCLC were presented during ASCO Annual Meeting 2015.[32] Cabozantinib, co-targeting angiogenesis and MET signaling plus erlotinib showed statistically significant improvement in both OS and PFS compared with erlotinib alone. Indeed, this trail was the only one of all included trials demonstrating overall survival benefits from combining therapy. Interestingly, our subset analysis based on different signaling pathways, involving 2,095 patients in anti-angiogenesis arm and 2,158 patients in anti-MET arm, suggested significant PFS improvement in patients treated with combined targeted agents including anti-angiogenesis (sorafenib, bevacizumab, pazopanib, sunitinib) and antiMET (tivantinib, onartuzumab) targeted agents.

It should be noted that our analysis was limited to the use of individual patient data. All the outcome estimates were taken from published data, which tended to overestimate treatments effects. Furthermore, 10 of the 24 included RCTs were marked with "open-label" and the performance bias was assessed as "high risk", which may decrease the quality of our meta-analysis.

Notably, according to NCCN Guidelines Version 2.2017, the standard of care in NSCLC now is to select patients based on their EGFR or $A L K$ status. As for patients with EGFR mutation or ALK rearrangement, several targeted drugs are recommended as first line

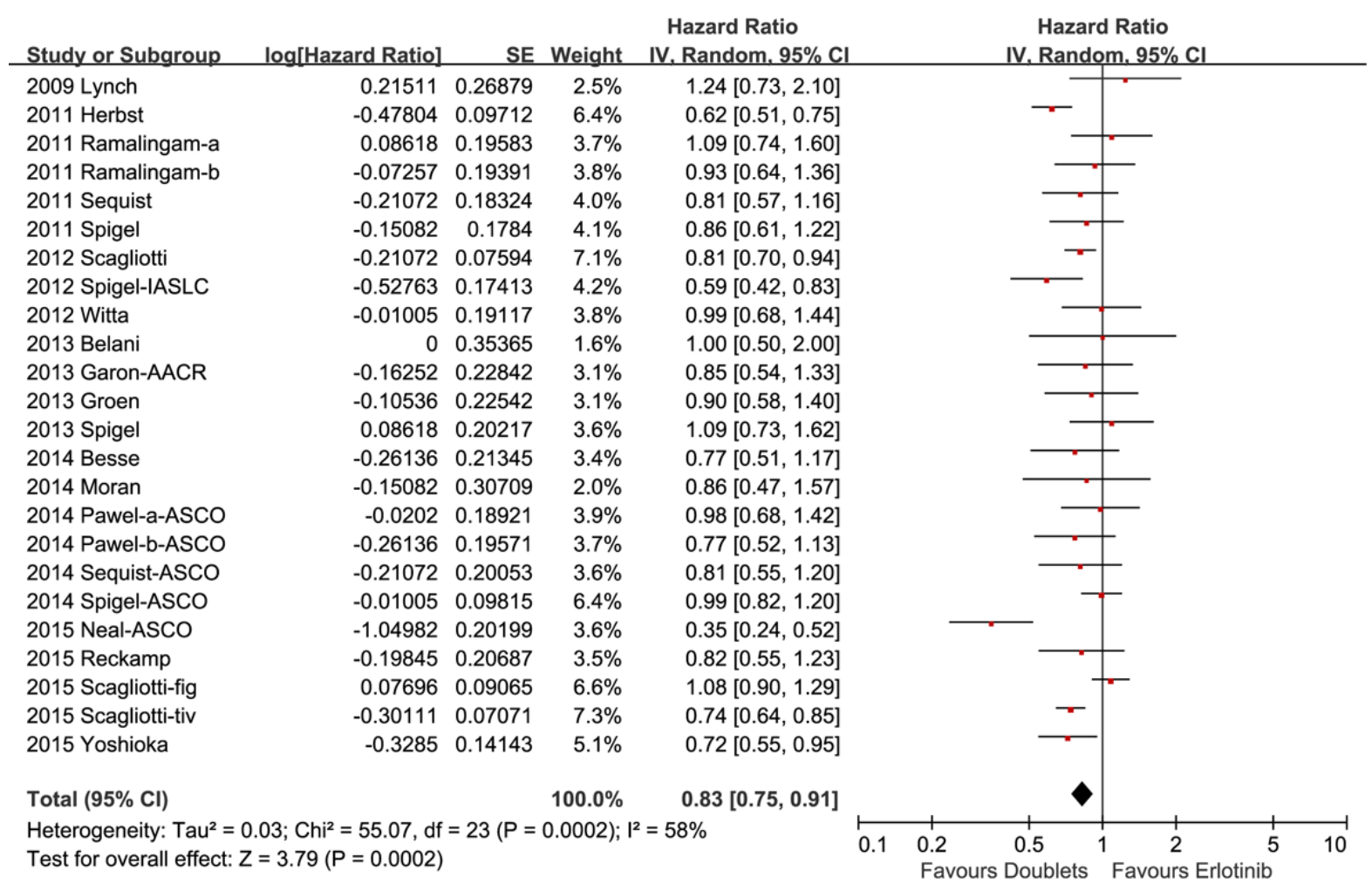

Figure 3: Forest plots for progression-free disease. 
choose. Chemotherapy is an first option for EGFR or $A L K$ negative patients. Therefore, RCTs studying erlotinib versus doublets targeted therapy are recommended being conducted in first-line setting. However, according to our update searching in PubMed database (February 5, 2017), there were only two articles reporting the efficacy of erlotinib compared to doublets in chemotherapynative patients (no additional studies based in second-line therapy were found). One is an open-label randomized phase II study compared the combination of erlotinib and bevacizumab versus erlotinib alone in patients with non-squamous NSCLC harboring EGFR mutations in first-line setting.[40] The addition of bevacizumab to erlotinib conferred a significant improvement in PFS. Another investigating erlotinib plus Linsitinib (an IGF-1R inhibitor) or placebo in chemotherapy-naive patients. [41] Considering the limited number of relevant studies in firstline setting, our meta-analysis which seems lagging in the contemporary management of NSCLC is actually of great referential value in assessing efficacy of erlotinib versus doublets in first-line therapy. Future clinical studies should be designed based on the actual data in our meta-analysis.

From this analysis, we conclude that erlotinib combined with additional targeted agent, especially anti-angiogenesis and anti-MET agent, could provide superior clinical benefit to patients with previously treated advanced NSCLC. The efficacy of combination therapy for particular selected populations, such as EGFR wildtype population, need further investigation. The absence of a biomarker to identify sensitive populations is a major hurdle for optimal utilization.

\section{MATERIALS AND METHODS}

\section{Protocol}

This review was conducted and reported according to the Preferred Reporting Items for Systematic Reviews and Meta-Analysis (PRISMA) Statement issued in 2009. No ethical approval and patient consent are required as all analysis were based on previous published studies.

\section{Search strategy}

A comprehensive and systematic search of the electronic databases (PubMed, Embase, and Cochrane) for studies published between inception and February 2, 2016 was conducted. Applicable terms, such as "erlotinib", "NSCLC", "combin*" were used in the literature search with the filter "randomized control trial". Relevant abstracts were searched and retrieved from American Society of Clinical Oncology (ASCO) databases.

\section{Study eligibility}

Studies investigating combining molecular targeted therapy based on erlotinib versus erlotinib plus placebo or erlotinib alone in patients with advanced NSCLC (stage IV or IIIB) were eligible for inclusion. Studies that satisfied all the following criteria were included: (i) patients with

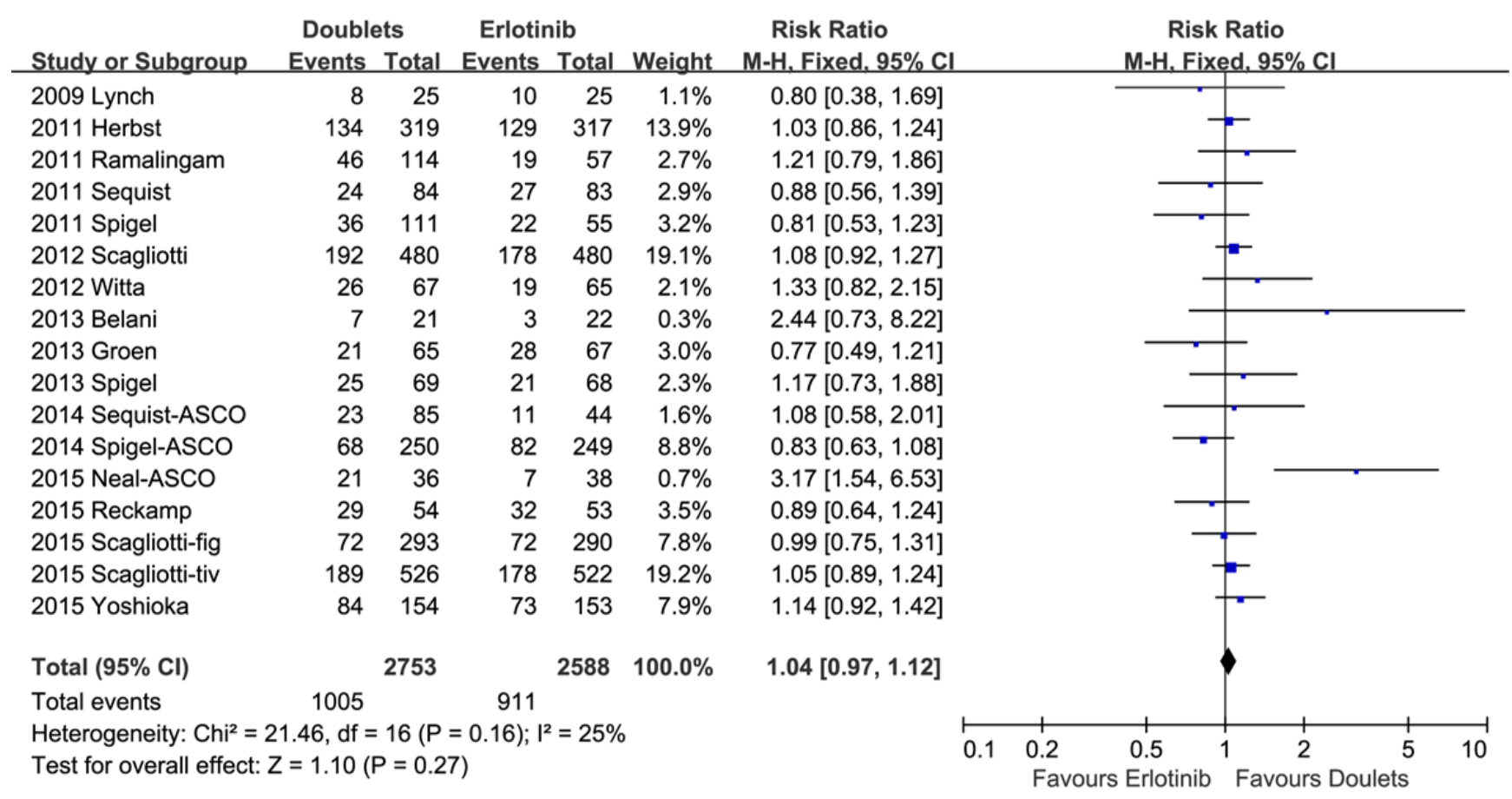

Figure 4: Forest plots for 1-year survival rate. 
histologically or cytologically confirmed stage IIIB or stage IV NSCLC and previously treated with at least one chemotherapy; (ii) assessing efficacy (and safety) profile of erlotinib-doublet targeted therapy versus single-agent erlotinib; (iii) phase II/III randomized controlled trials; (iv) at least one of the following outcome measures was

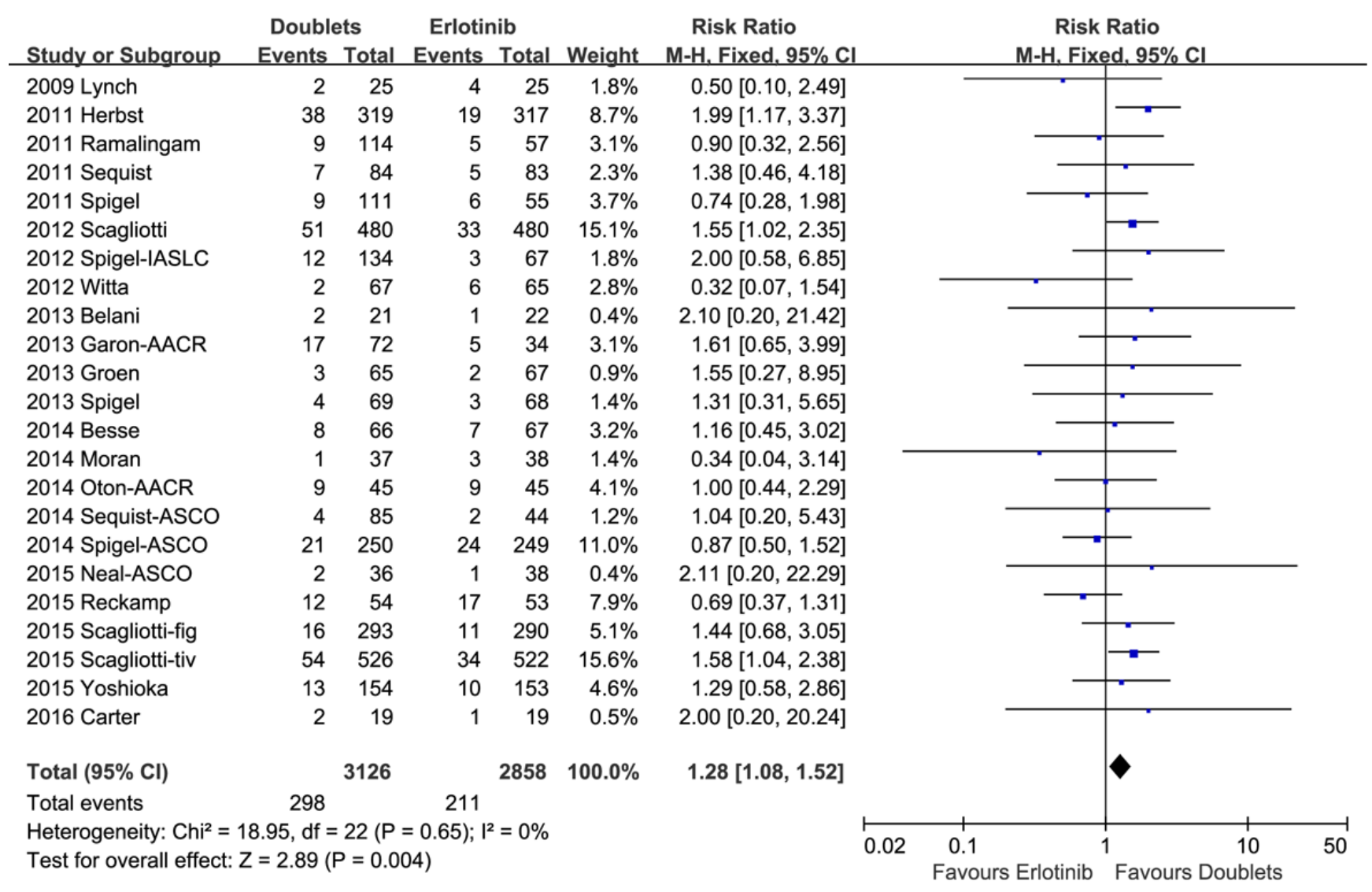

Figure 5: Forest plots for objective response rate.

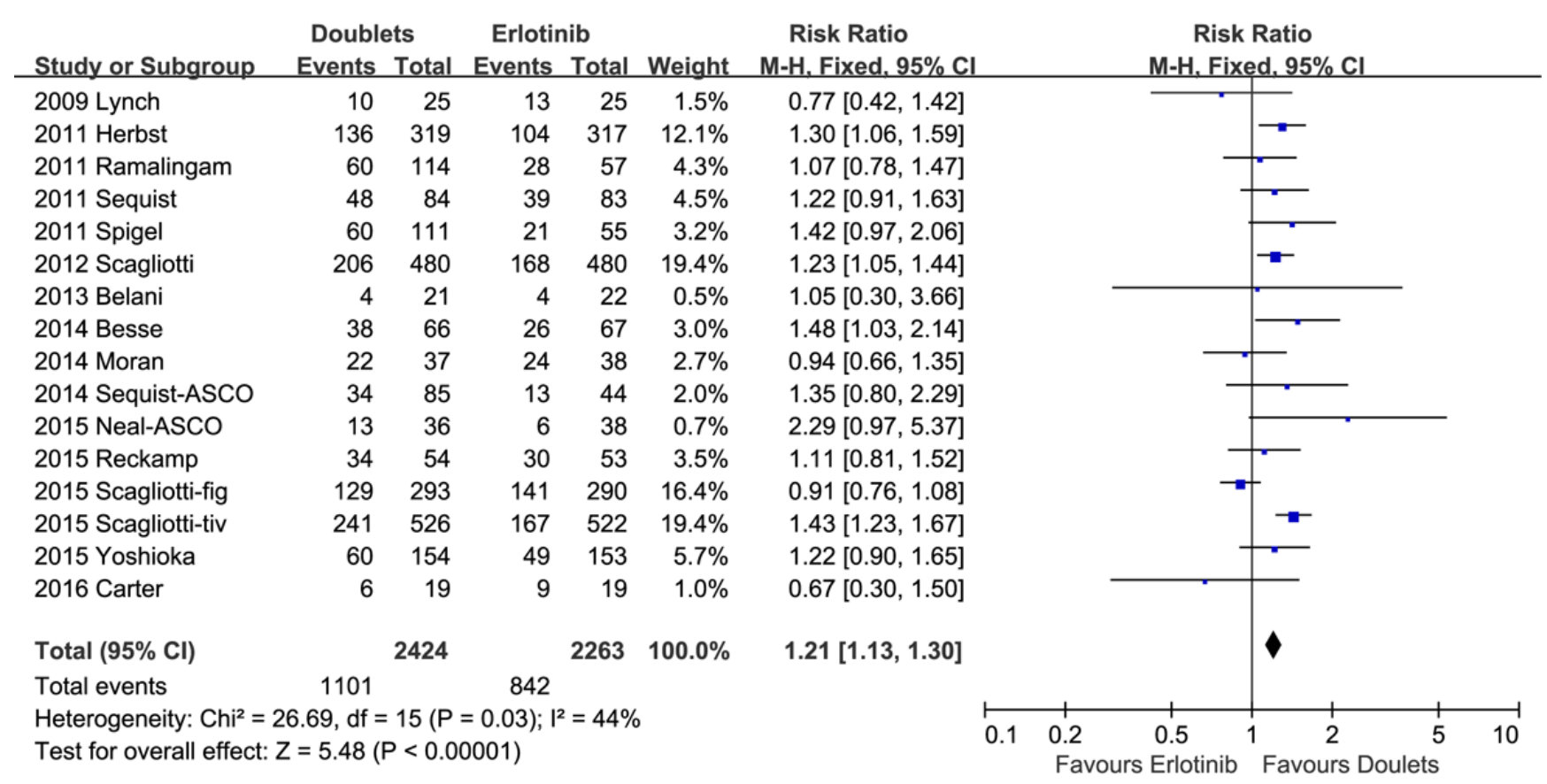

Figure 6: Forest plots for disease control rate. 
extractable in an analyzable form: overall survival (OS), progression-free survival (PFS), objective response rate (ORR), disease control rate (DCR) or 1-year survival rate (SR).

The exclusion criteria were as follows: (i) duplicate reports; (ii) review articles; (iii) case reports; (iv) phase I and single-arm phase II trials owing to a lack of control groups; (v) ongoing studies; (vi) studies investigating targeted therapy as first-line treatment; (vii) studies not within the field of interest of this study.

\section{Data extraction}

Data extraction from eligible studies were performed independently by two reviewers and disagreements were resolved by discussion and consensus with a third reviewer. The following information was extracted: the first author, year, trial phase, interventions, targeted pathways, number of subjects, median age, the percentage of female, smoking history, histology, ECOG performance status, stage, prior chemotherapy regimens, median OS, median PFS, ORR, DCR, 1-year SR, and the hazard ratio (HR) along with 95\% confidence interval (CI) for the comparison of OS or PFS of erlotinib-based doubletstreated patients with that of patients receiving erlotinib alone. If the HR and 95\% CI was not directly reported in the article, an estimation from the survival curve was made using Tierney's method.[42]

\section{Assessment of risk of bias in included studies}

The methodological quality of RCTs was assessed using the risk of bias tool following the Cochrane Collaboration guidelines. Seven domains were employed for this part including random sequence generation, allocation concealment, blinding of participants, personnel or outcome assessment, incomplete outcome data, selective reporting and other sources of bias.

\section{Statistical analysis}

The pooled HR for time-to-event outcomes (OS, PFS) and pooled relative risk (RR) for dichotomous data (ORR, DCR, and 1-year SR was calculated using the Review Manager 5.3 software statistical software. Heterogeneity assessed with the inconsistency statistic $\left(I^{2}\right)$ was interpreted as follows: $I^{2}=0 \%$ indicates no heterogeneity, $0 \%<I^{2}<25 \%$ indicates the least heterogeneity, $25 \% \leq I^{2}<50 \%$ indicates mild heterogeneity, $50 \% \leq I^{2}<75 \%$ indicates moderate heterogeneity, and $75 \% \leq I^{2}$ indicates strong heterogeneity. [43] We employed a random-effects model in case of the existence of moderate or strong heterogeneity $\left(I^{2} \geq\right.$ $50 \%$ ). Otherwise, a fixed-effects model was used. We pooled time-to event data using inverse variance method and dichotomous data with Mantel-Haenszel method. Subgroup analysis was performed according to phases of trials, targeted signaling pathways, EGFR-status and $K R A S$-status. $p$ values $<0.05$ were regarded as being statistically significant for all included studies. Publication bias was evaluated according to Begg's and Egger's test using the STATA 12.0 software statistical software.

\section{ACKNOWLEDGMENTS AND FUNDING}

Funded by the National Natural Science Foundation of China (No. 81570078), the Natural Science Foundation of Jiangsu Province (No. BK20161386).

\section{CONFLICTS OF INTEREST}

The authors have declared no conflicts of interest.

\section{REFERENCES}

1. WHO. Cancer fact sheet no. 297. February 2015. http:// www.who.int/mediacentre/factsheets/fs297/en/

2. Chen W, Zheng R, Baade PD, Zhang S, Zeng H, Bray F, Jemal A, Yu XQ, He J. Cancer statistics in China, 2015. Ca Cancer J Clin. 2016; 66:115-132.

3. Schiller JH, Harrington D, Belani CP, Langer C, Sandler A, Krook J, Zhu J, Johnson DH. Comparison of four chemotherapy regimens for advanced non-small-cell lung cancer. N Engl J Med. 2002; 346:92-98.

4. Ettinger DS, Akerley W, Bepler G, Blum MG, Chang A, Cheney RT, Chirieac LR, D'Amico TA, Demmy TL, Ganti AK, Govindan R, Grannis FJ, Jahan T, et al. Non-small cell lung cancer. J Natl Compr Canc Netw. 2010; 8:740-801.

5. Shepherd FA, Rodrigues PJ, Ciuleanu T, Tan EH, Hirsh V, Thongprasert S, Campos D, Maoleekoonpiroj S, Smylie M, Martins R, van Kooten M, Dediu M, Findlay B, et al. Erlotinib in previously treated non-small-cell lung cancer. N Engl J Med. 2005; 353:123-132.

6. Yap TA, Omlin A, de Bono JS. Development of therapeutic combinations targeting major cancer signaling pathways. J Clin Oncol. 2013; 31:1592-1605.

7. Bluthgen M, Besse B. Second-line combination therapies in nonsmall cell lung cancer without known driver mutations. Eur Respir Rev. 2015; 24:582-593.

8. Chong CR, Jänne PA. The quest to overcome resistance to EGFR-targeted therapies in cancer. Nat Med. 2013; 19:1389-1400.

9. Lynch TJ, Fenton D, Hirsh V, Bodkin D, Middleman EL, Chiappori A, Halmos B, Favis R, Liu H, Trepicchio WL, Eton O, Shepherd FA. A randomized phase 2 study of erlotinib alone and in combination with bortezomib in previously treated advanced non-small cell lung cancer. J 
Thorac Oncol. 2009; 4:1002-1009.

10. Spigel DR, Burris HR, Greco FA, Shipley DL, Friedman EK, Waterhouse DM, Whorf RC, Mitchell RB, Daniel DB, Zangmeister J, Bass JD, Hainsworth JD. Randomized, double-blind, placebo-controlled, phase II trial of sorafenib and erlotinib or erlotinib alone in previously treated advanced non-small-cell lung cancer. J Clin Oncol. 2011; 29:2582-2589.

11. Sequist LV, Pawel JV, Garmey EG, Akerley WL, Brugger W, Ferrari D, Chen Y, Costa DB, Gerber DE, Orlov S, Ramlau R, Arthur S, Gorbachevsky I, et al. Randomized phase II study of erlotinib plus tivantinib versus erlotinib plus placebo in previously treated non-small-cell lung cancer. J Clin Oncol. 2011; 29:3307-3315.

12. Herbst RS, Ansari R, Bustin F, Flynn P, Hart L, Otterson GA, Vlahovic G, Soh CH, O'Connor P, Hainsworth J. Efficacy of bevacizumab plus erlotinib versus erlotinib alone in advanced non-small-cell lung cancer after failure of standard first-line chemotherapy (BeTa): a double-blind, placebo-controlled, phase 3 trial. Lancet. 2011; 377:18461854.

13. Ramalingam SS, Spigel DR, Chen D, Steins MB, Engelman JA, Schneider CP, Novello S, Eberhardt WE, Crino L, Habben K, Liu L, Janne PA, Brownstein CM, et al. Randomized phase II study of erlotinib in combination with placebo or R1507, a monoclonal antibody to insulin-like growth factor-1 receptor, for advanced-stage non-small-cell lung cancer. J Clin Oncol. 2011; 29:4574-4580.

14. Scagliotti GV, Krzakowski M, Szczesna A, Strausz J, Makhson A, Reck M, Wierzbicki RF, Albert I, Thomas M, Miziara JEA, Papai ZS, Karaseva N, Thongprasert S, et al. Sunitinib plus erlotinib versus placebo plus erlotinib in patients with previously treated advanced non-small-cell lung cancer: A phase III trial. J Clin Oncol. 2012; 30:20702078.

15. Witta SE, Jotte RM, Konduri K, Neubauer MA, Spira AI, Ruxer RL, Varella-Garcia M, Bunn JPA, Hirsch FR. Randomized phase II trial of erlotinib with and without entinostat in patients with advanced non-small-cell lung cancer who progressed on prior chemotherapy. J Clin Oncol. 2012; 30:2248-2255.

16. Belani CP, Nemunaitis JJ, Chachoua A, Eisenberg PD, Raez LE, Cuevas JD, Mather CB, Benner RJ, Meech SJ. Phase 2 trial of erlotinib with or without PF-3512676 (CPG 7909, a Toll-like receptor 9 agonist) in patients with advanced recurrent EGFR-positive non-small cell lung cancer. Cancer Biol Ther. 2013; 14:557-563.

17. Spigel DR, Ervin TJ, Ramlau RA, Daniel DB, Goldschmidt JH, Blumenschein GR, Krzakowski MJ, Robinet G, Godbert B, Barlesi F, Govindan R, Patel T, Orlov SV, et al. Randomized phase II trial of Onartuzumab in combination with erlotinib in patients with advanced non-small-cell lung cancer. J Clin Oncol. 2013; 31:4105-4114.

18. Groen HJ, Socinski MA, Grossi F, Juhasz E, Gridelli C, Baas P, Butts CA, Chmielowska E, Usari T, Selaru P,
Harmon C, Williams JA, Gao F, et al. A randomized, double-blind, phase II study of erlotinib with or without sunitinib for the second-line treatment of metastatic nonsmall-cell lung cancer (NSCLC). Ann Oncol. 2013; 24:2382-2389.

19. Besse B, Leighl N, Bennouna J, Papadimitrakopoulou VA, Blais N, Traynor AM, Soria JC, Gogov S, Miller N, Jehl $\mathrm{V}$, Johnson BE. Phase II study of everolimus-erlotinib in previously treated patients with advanced non-small-cell lung cancer. Ann Oncol. 2014; 25:409-415.

20. Moran T, Felip E, Keedy V, Borghaei H, Shepherd FA, Insa A, Brown H, Fitzgerald T, Sathyanarayanan S, Reilly JF, Mauro D, Hsu K, Yan L, et al. Activity of dalotuzumab, a selective anti-IGF1R antibody, in combination with erlotinib in unselected patients with Non-small-cell lung cancer: A phase I/II randomized trial. Exp Hematol Oncol. 2014; 3:26.

21. Scagliotti GV, Bondarenko I, Blackhall F, Barlesi F, Hsia TC, Jassem J, Milanowski J, Popat S, Sanchez-Torres JM, Novello S, Benner RJ, Green S, Molpus K, et al. Randomized, phase III trial of figitumumab in combination with erlotinib versus erlotinib alone in patients with nonadenocarcinoma nonsmall-cell lung cancer. Ann Oncol. 2015; 26:497-504.

22. Scagliotti G, Pawel JV, Novello S, Ramlau R, Favaretto A, Barlesi F, Akerley W, Orlov S, Santoro A, Spigel D, Hirsh V, Shepherd FA, Sequist LV, et al. Phase III Multinational, Randomized, Double-Blind, Placebo-Controlled Study of Tivantinib (ARQ 197) Plus Erlotinib Versus Erlotinib Alone in Previously Treated Patients With Locally Advanced or Metastatic Nonsquamous Non-Small-Cell Lung Cancer. J Clin Oncol. 2015; 33:2667-2674.

23. Reckamp KL, Koczywas M, Cristea MC, Dowell JE, Wang HJ, Gardner BK, Milne GL, Figlin RA, Fishbein MC, Elashoff RM, Dubinett SM. Randomized phase 2 trial of erlotinib in combination with high-dose celecoxib or placebo in patients with advanced non-small cell lung cancer. Cancer. 2015; 121:3298-3306.

24. Yoshioka H, Azuma K, Yamamoto N, Takahashi T, Nishio M, Katakami N, Ahn MJ, Hirashima T, Maemondo M, Kim SW, Kurosaki M, Akinaga S, Park K, et al. A randomized, double-blind, placebo-controlled, phase III trial of erlotinib with or without a c-Met inhibitor tivantinib (ARQ 197) in Asian patients with previously treated stage IIIB/IV nonsquamous nonsmall-cell lung cancer harboring wildtype epidermal growth factor receptor (ATTENTION study). Ann Oncol. 2015; 26:2066-2072.

25. Carter CA, Rajan A, Keen C, Szabo E, Khozin S, Thomas A, Brzezniak C, Guha U, Doyle LA, Steinberg SM, Xi L, Raffeld M, Tomita Y, et al. Selumetinib with and without erlotinib in KRAS mutant and KRAS wild-type advanced nonsmall-cell lung cancer. Ann Oncol. 2016; 27:693-699.

26. Spigel D, Burris HA, Greco FA, Shih KC, Lipman AJ, Flora DB, Daniel DB, Waterhouse DM, Haymach JV, Hainsworth JD. A randomized phase II study of pazopanib 
or placebo in combination with erlotinib in patients with advanced non-small-cell lung cancer. J Thorac Oncol. 2012 (Suppl 9); 7:S203-S335. http://dx.doi.org/10.1097/ JTO.0b013e318269fc07.

27. Garon EB, Siegfried JM, Dubinett SM, Elashoff RM, Park DJ, Parikh RJ, Patel R, Hu EH, Reckamp KL, Adams B, Martinez D, Wang HJ, Kabbinavar F, et al. Result of TORI L-03, a randomized, multicenter phase II clinical trial of erlotinib $(\mathrm{E})$ or $\mathrm{E}+$ fulvestrant $(\mathrm{F})$ in previously treated advanced non-small cell lung cancer (NSCLC). Cancer Res. 2013 (Suppl 8); 73:4664. http://dx.doi.org/10.1158/15387445.AM2013-4664.

28. Oton AB, Cho BC, Ahn MJ, Kim SW, Subramanian K, Desai C, Shuster D, Goldberg T, Zahir H, Dutta D, Chen S, Von Roemeling R, Von Pawel J. A randomized, openlabel phase 2 study of efatutazone and erlotinib as second-or third-line therapy for non-small cell lung cancer (NSCLC). Cancer Res. 2014 (Suppl 19); 74:CT311. http://dx.doi. org/10.1158/1538-7445.AM2014-CT311.

29. Spigel DR, Edelman MJ, O'Byrne K, Paz-Ares L, Shames DS, Yu W, Paton VE, Mok T. Onartuzumab plus erlotinib versus erlotinib in previously treated stage IIIb or IV NSCLC: Results from the pivotal phase III randomized, multicenter, placebo-controlled METLung (OAM4971g) global trial. J Clin Oncol. 2014 (Suppl 15); 32:8000.

30. Pawel JV, Tseng J, Dediu M, Schumann C, Moritz B, Mendell-Harary J, Jin X, Feng W, Copigneaux C, Beckman RA. Phase 2 HERALD study of patritumab (P) with erlotinib (E) in advanced NSCLC subjects (SBJs). J Clin Oncol. 2014 (Suppl 15); 32:8045.

31. Sequist L, Lopez-Chavez A, Doebele RC, Gray JE, Harb WA, Modiano MR, Jackman DM, Baggstrom MQ, Atmaca A, Felip E, Provencio M, Cobo M, Kripas CJ, et al. A randomized phase 2 trial of MM-121, a fully human monoclonal antibody targeting ErbB3, in combination with erlotinib in EGFR wild-type NSCLC patients. J Clin Oncol. 2014 (Suppl 15); 32:8051.

32. Neal JW, Dahlberg SE, Wakelee HA, Aisner SC, Bowden M, Carbone DP, Ramalingam SS. Cabozantinib (C), erlotinib $(\mathrm{E})$ or the combination $(\mathrm{E}+\mathrm{C})$ as second-or thirdline therapy in patients with EGFR wild-type (wt) nonsmall cell lung cancer (NSCLC): A randomized phase 2 trial of the ECOGACRIN Cancer Research Group (E1512). J Clin Oncol. 2015 (Suppl 15); 33:8003.

33. Blumenthal GM, Karuri SW, Zhang H, Zhang L, Khozin S, Kazandjian D, Tang S, Sridhara R, Keegan P, Pazdur R. Overall response rate, progression-free survival, and overall survival with targeted and standard therapies in advanced non-small-cell lung cancer: US Food and Drug Administration trial-level and patient-level analyses. J Clin Oncol. 2015; 33:1008-1014.

34. Pan G, Ke S, Zhao J. Comparison of the efficacy and safety of single-agent erlotinib and doublet molecular targeted agents based on erlotinib in advanced non-small cell lung cancer (NSCLC): a systematic review and meta-analysis.
Targeted Oncology. 2013; 8:107-116.

35. Qi WX, Wang Q, Jiang YL, Sun YJ, Tang LN, He AN, Min DL, Lin F, Shen Z, Yao Y. Overall survival benefits for combining targeted therapy as second-line treatment for advanced non-small-cell-lung cancer: a meta-analysis of published data. PLoS One. 2013; 8:e55637.

36. Birchmeier C, Birchmeier W, Gherardi E, Vande WG. Met, metastasis, motility and more. Nat Rev Mol Cell Biol. 2003; 4:915-925.

37. Go H, Jeon YK, Park HJ, Sung SW, Seo JW, Chung DH. High MET gene copy number leads to shorter survival in patients with non-small cell lung cancer. J Thorac Oncol. 2010; 5:305-313.

38. Engelman JA, Zejnullahu K, Mitsudomi T, Song Y, Hyland C, Park JO, Lindeman N, Gale CM, Zhao X, Christensen J, Kosaka T, Holmes AJ, Rogers AM, et al. MET amplification leads to gefitinib resistance in lung cancer by activating ERBB3 signaling. Science. 2007; 316:10391043.

39. Sandler A, Gray R, Perry MC, Brahmer J, Schiller JH, Dowlati A, Lilenbaum R, Johnson DH. Paclitaxelcarboplatin alone or with bevacizumab for non-small-cell lung cancer. N Engl J Med. 2006; 355:2542-2550.

40. Seto T, Kato T, Nishio M, Goto K, Atagi S, Hosomi Y, Yamamoto N, Hida T, Maemondo M, Nakagawa K, Nagase S, Okamoto I, Yamanaka T, et al. Erlotinib alone or with bevacizumab as first-line therapy in patients with advanced non-squamous non-small-cell lung cancer harbouring EGFR mutations (JO25567): an open-label, randomised, multicentre, phase 2 study. Lancet Oncol. 2014; 15:12361244.

41. Leighl NB, Rizvi NA, de Lima LJ, Arpornwirat W, Rudin CM, Chiappori AA, Ahn MJ, Chow LQ, Bazhenova L, Dechaphunkul A, Sunpaweravong P, Eaton K, Chen J, et al. Phase 2 Study of Erlotinib in Combination With Linsitinib (OSI-906) or Placebo in Chemotherapy-Naive Patients With Non-Small-Cell Lung Cancer and Activating Epidermal Growth Factor Receptor Mutations. Clin Lung Cancer. 2017; 18:34-42.

42. Tierney JF, Stewart LA, Ghersi D, Burdett S, Sydes MR. Practical methods for incorporating summary time-to-event data into meta-analysis. Trials. 2007; 8:16.

43. Higgins JP, Thompson SG, Deeks JJ, Altman DG. Measuring inconsistency in meta-analyses. Brit Med J. $2003 ; 327: 557-560$. 